\title{
6
}

\section{CLINICAL COGNITIVE SCIENCE: APPLYING QUANTITATIVE MODELS OF COGNITIVE PROCESSING TO EXAMINE COGNITIVE ASPECTS OF PSYCHOPATHOLOGY}

TERESA A. TREAT, RICHARD M. MCFALL, RICHARD ]. VIKEN, JOHN K. KRUSCHKE, ROBERT M. NOSOFSKY, AND SHIRLEY S. WANG

Numerous theoretical approaches implicate a role for altered cognitive processing in the development, maintenance, and treatment of a wide range of clinically relevant behaviors. Clinical scientists have been slow, however, to capitalize on the wealth of contemporary theoretical, measurement, and analytical models of cognitive processing when constructing, evaluating, and extending these theoretical approaches to psychopathology (MacLeod, 1993; McFall \& Townsend, 1998; McFall, Treat, \& Viken, 1998). To date, the translation of cognitive science models to clinical science has occurred primarily at the measurement or task level, leading to an impoverished understanding and utilization of the multifaceted complexity of the relevant theoretical constructs, such as attention, classification, memory, and learning. As a result, clinical scientists have tended to collapse, or ignore the distinctions between, theoretical and measurement models of cognitive 
processes and to reify cognitive tasks as the theoretical constructs themselves (e.g., the emotional Stroop and dot-probe paradigms often are assumed to "be" attention and are pulled off the shelf whenever researchers need a measure of attention). Clinical scientists' failure to translate many of cognitive scientists' theoretically grounded analytical models also slows the advancement of clinical cognitive science. Fitting formal analytical models, or process models, not only forces clinical researchers to quantify and evaluate rigorously their theoretical assumptions about the operation of the cognitive processes of interest but also enhances their understanding of the mechanisms hypothesized to underlie variation in observed performance on cognitive-processing tasks.

The overarching aims of this chapter are to introduce a unified class of theoretical, measurement, and analytical models that can be used to examine research questions about clinically relevant cognitive processing and to illustrate the generalizability and applicability of these cognitive science models to more real-world research questions. This well-established class of models treats participants' perceptual organizations of stimuli as a primitive on which other processes - such as classification, memory, and learning - operate (for evidence of the validity of these models, see Kruschke, 1992; Kruschke \& Johansen, 1999; Nosofsky, 1991, 1992a, 1992b). Formal process models specify mathematically the theorized links among these interrelated processes and afford rigorous examination of the mechanisms underlying task performance. Thus, this approach not only accounts simultaneously for the operation of multiple cognitive processes but also specifies well-integrated theoretical, measurement, and analytical models of these processes.

To examine the feasibility and utility of using this clinical cognitive approach, we deliberately have examined it in parallel across multiple areas of psychopathology, with a primary focus on problematic eating patterns and sexually aggressive behaviors. Theoretical approaches within both domains increasingly have focused on the role of information-processing patterns in the etiology and maintenance of these behaviors and in the development of prevention and intervention strategies (e.g., McFall, 1990; Schewe \& O'Donohue, 1993; Vitousek, 1996; Ward, Hudson, Johnston, \& Marshall, 1997; Wilson, 1999). In this chapter, we focus on the use of cognitive science methods to characterize individual differences in men's processing of women's facial affect and physical appearance, with implications for our understanding of sexual aggression, and individual differences in women's processing of other women's facial affect and body size, with implications for our understanding of eating disorders. We open this chapter with a section on stimulus-construction issues, given their centrality to the utility and validity of the approach. 
Cognitive scientists commonly rely on simple, artificial stimulus sets that vary along a finite number of readily identifiable dimensions that are perceived in a relatively uniform fashion across participants (e.g., rectangles that vary in height and width, or color patches that vary in hue and saturation). These well-controlled and decontextualized stimulus sets facilitate investigation of cognitive scientists' research questions about process models of normative information processing. Clinical scientists, in contrast, more commonly are interested in characterizing individual differences in the processing of much more complex, socially relevant stimuli that vary along numerous dimensions. As a result, the development of an appropriate stimulus set is a critical and time-consuming process for clinical researchers.

Stimulus development begins by specifying explicitly a small number of dimensions of theoretical interest. In our experience, the incorporation of more than three or four systematically manipulated dimensions in a stimulus set becomes unwieldy, given the need to include multiple stimuli per dimension. Within the domain of eating disorders, for example, we have sought to evaluate questions about individual differences in women's processing of information about other women's body size and facial affect (Viken, Treat, Nosofky, McFall, \& Palmeri, 2002). The stimulus set ideally should include both dimensions that are predicted to draw more attention from the clinical population of interest and dimensions that are predicted to draw equal or less attention from the clinical population. This strategy facilitates the examination of alternative explanations that are plausible but less theoretically interesting. For example, demonstrating that highsymptom women, relative to low-symptom women, attend both significantly more to body size and significantly less to facial affect is inconsistent with group differences in intelligence, motivation, level of perfectionism, and so on. Our use of formal process models to evaluate our hypotheses also facilitates the rigorous evaluation of competing models (cf. Knight $\&$ Silverstein, 2001; Neufeld, Vollick, Carter, Boksman, \& Jetté, 2002).

The second step in stimulus development entails selection of an appropriate presentation medium. To date, we have relied primarily on photo stimulus sets, but alternatives include words or phrases describing objects or people, situations described in brief vignettes, audio or video clips of interpersonal interactions, and combinations of these possibilities (e.g., presenting photos with accompanying short text). The potential media vary in terms of the extent to which they provide ecologically valid representation of relevant stimulus features and the extent to which they afford control of irrelevant stimulus features. Ideally, the selected medium allows researchers to maximize variability along the dimensions of primary theoretical 
interest in an ecologically valid fashion and to minimize or eliminate variability along theoretically irrelevant dimensions.

Next, researchers develop a large potential stimulus pool that incorporates sufficient and representative variability along the dimensions of primary theoretical interest and minimizes or eliminates variability along unimportant dimensions. Thus, when developing the photo stimulus set for our eating-disorders research program, we photographed numerous college-age volunteers as they displayed happy, neutral, and sad facial expressions. The naturally occurring variability in body size among models was less pronounced than we had hoped, so we digitally altered the photographs to increase variability along this dimension. Variability in other aspects of appearance was of less interest theoretically but likely to draw attention, so we asked all volunteers to wear similar outfits, to remove makeup, and to pull their hair back from their faces. Photographs also were taken in front of a fixed background and under standard lighting conditions, in an effort to eliminate variability along these theoretically uninteresting dimensions.

The fourth step in stimulus development involves the collection of normative data along dimensions of potential relevance to participants' processing of the stimulus set and the selection of a preliminary stimulus set. Thus, we asked a sample of undergraduate women to make explicit ratings of the models' body size, facial affect, attractiveness, friendliness, and self-esteem along 10-point scales. The average ratings on these dimensions provided normative values for the stimuli along the body-size and affect dimensions. When selecting a subset of the stimuli for use in cognitiveprocessing studies, we retained only one photo per model and endeavored to include sufficient and representative variability both along and across the two theoretical dimensions of greatest interest to us (i.e., we included women in each of the four quadrants of the desired two-dimensional stimulus space). We also selected stimuli that minimized the correlation between the normative ratings of the two dimensions, because we were interested in characterizing the processing of the two dimensions as independently as possible.

The final step necessitates conducting a multidimensional scaling (MDS) study to evaluate four things: (a) whether the population of interest attends to the dimensions of theoretical interest when processing the stimuli (i.e., whether the psychological dimensions correspond to the physical dimensions); (b) whether participants attend too much to irrelevant dimensions, necessitating a retooling of the stimulus set; (c) whether the two dimensions are perceived relatively independently; and (d) whether individual differences in attention to the dimensions emerge. In the next section, we describe this process. 
Overall, the stimulus development process aims to construct a set of stimuli that both represents the real-world variability along the dimensions of greatest theoretical interest and controls or constrains the real-world variability along the dimensions of least theoretical interest. This frequently necessitates construction of a stimulus set from scratch, because preexisting stimuli often fail to meet these criteria. In our experience, this process is time consuming but invaluable, because the validity and precision of all inferences about attention to, classification of, memory for, and learning about the theoretical dimensions of interest depend to a significant extent on the adequacy of the stimulus set.

\section{PERCEPTUAL ORGANIZATION}

Perceptual organization (PO) refers to the representation and organization of incoming stimuli in terms of their perceived similarity and dissimilarity. Numerous theoretical perspectives in clinical psychology are consistent with the perspective that psychopathology is determined or maintained partly by features of participants' POs, such as attention to stimulus dimensions, the perceived association between stimulus dimensions, and the organization of stimulus dimensions as discrete versus continuous (e.g., Beck, 1976; Kelly, 1955). The class of cognitive models of interest in this chapter treats participants' POs as representational bases for the operation of other higher order cognitive processes, such as classification, memory, and learning (Kruschke, 1992; Kruschke \& Johansen, 1999; Nosofsky, 1991, 1992a, 1992b). For example, the process models outlined in subsequent sections predict that participants (a) should classify a presented stimulus into a category containing the most similar members, (b) should recognize a presented stimulus as previously viewed when it is highly similar to previously stored stimuli, and (c) should learn a category structure based on a particular stimulus feature more quickly when stimuli in different categories are perceived to be very dissimilar and stimuli in the same category are perceived to be very similar.

PO frequently is assessed using a similarity-rating paradigm, in which participants judge the similarity of pairs of stimuli on a scale anchored by very different and very similar. Participants are told that there are no right or wrong answers and usually are encouraged to respond quickly with their first impression rather than to deliberate extensively about their decisions. This task provides a relatively implicit assessment of participants' POs, because it neither specifies the stimulus attributes of interest nor directs participants to attend to particular stimulus attributes. In an alternative paradigm, participants judge as quickly as possible whether presented pairs 
of stimuli are the same or different; in this case, either participants' judgments or their reaction times can be submitted for subsequent analysis (Nosofsky, 1992b). Given the distinctiveness and complexity of the stimuli of interest to clinical researchers, this latter paradigm may prove to be less useful in applied contexts.

MDS analyses of participants' similarity ratings provide a spatial representation of participants' PO or psychological space, in which the perceived similarity between two stimuli, $\delta_{i j}$, is modeled as a decreasing function of the distance, $d_{i j}$, between the perceived values of two stimuli, $x_{i}$ and $x_{i}$ (Davison, 1992; Treat et al., 2002). Thus, two stimuli that are judged to be very similar are scaled much closer in the psychological space than two stimuli that are judged to be very dissimilar (see Figure 6.1). The upper left-hand panel of Figure 6.1 presents the group psychological space of 24 photo stimuli that portray women who vary along facial-affect and bodysize dimensions. Stimuli A and B, which were judged to be very similar, are scaled close together. In contrast, both stimuli are judged to be very dissimilar to stimulus $\mathrm{C}$, which is scaled far away from stimuli A and B.

Metric scaling approaches assume that the function relating similarity and distance decreases linearly, whereas less restrictive nonmetric approaches assume only that this function decreases monotonically. Distances typically are computed using a Euclidean metric (i.e., $r=2$ in Equation 6.1) when the $M$ stimulus dimensions are processed more holistically, or integrally. In contrast, a city-block metric (i.e., $r=1$ ) is assumed when the $M$ stimulus dimensions are perceived more distinctively or separably (Nosofsky \& Palmeri, 1996; Shepard, 1964). In our experience, the correct metric for the more complex, ecologically valid stimulus sets of interest to clinical researchers often lies between these two extremes. Thus, it may prove useful for researchers to estimate this parameter rather than fixing it at either 1.0 or 2.0:

$$
d_{i j}=\left[\sum_{m=1}^{M}\left(x_{i m}-x_{j m}\right)^{r}\right]^{1 / r} .
$$

In the weighted MDS (WMDS) model, which also is known as the individual differences scaling (INDSCAL) model, individual differences in the $k$ participants' similarity ratings, $\delta_{i j k}$, are modeled as a decreasing function of interstimulus distances, $d_{i j k}$ (Carroll $\&$ Chang, 1970). These distances vary as a function of individual differences in the participant-specific weighting of the $M$ stimulus dimensions, $u_{m k}$, as specified in Equation 6.2:

$$
d_{i j k}=\left[\sum_{m=1}^{M} w_{m k}\left(x_{i m}-x_{j m}\right)^{r}\right]^{1 / r} .
$$




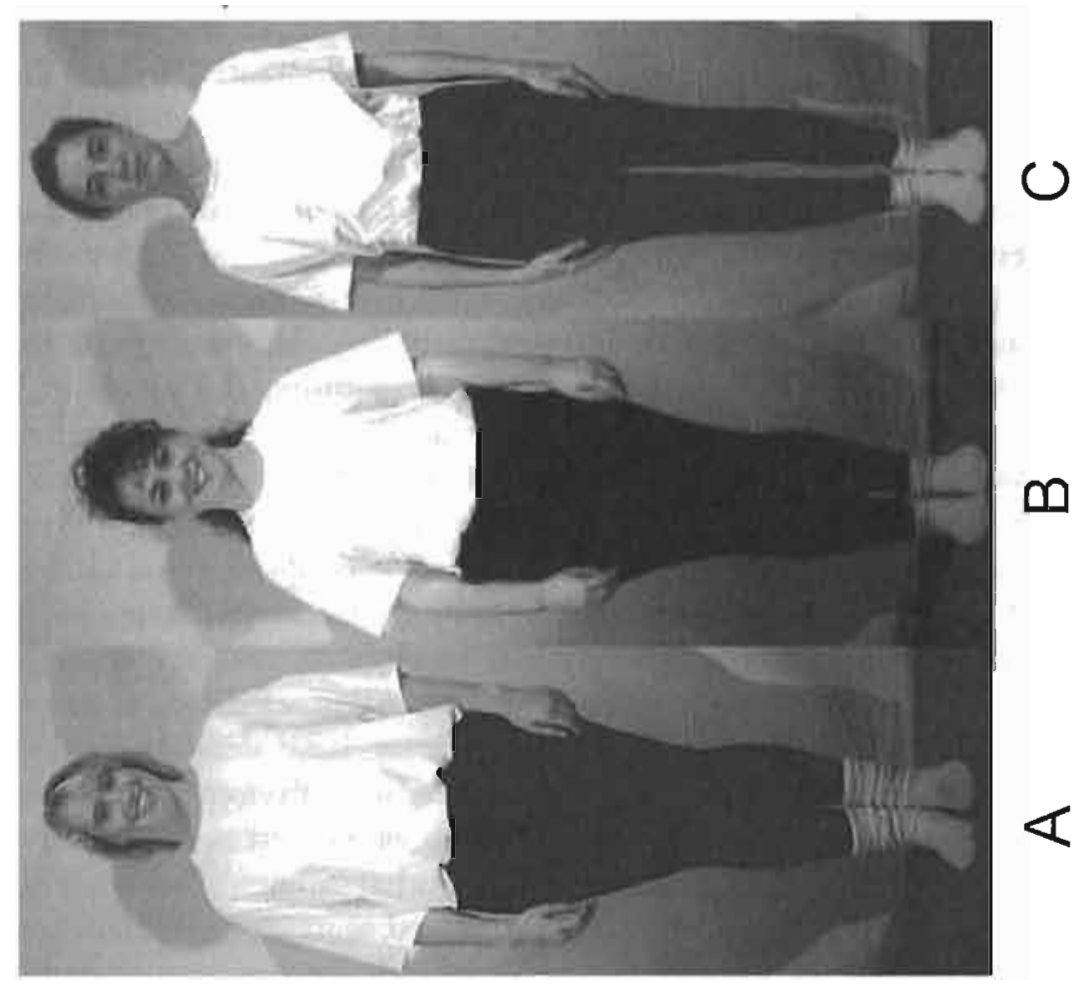

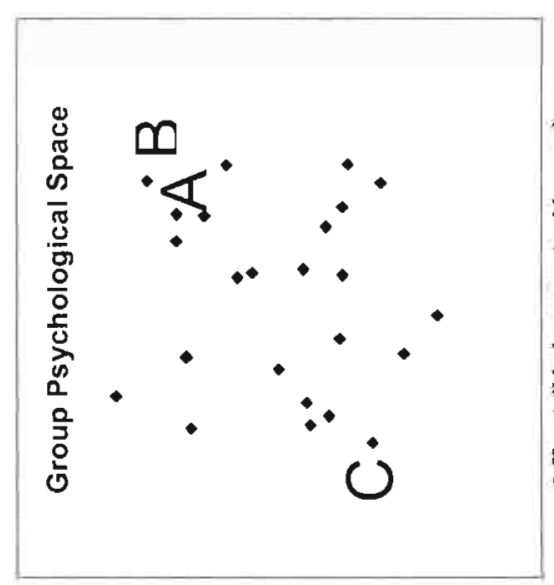

(К^еән 아 746!) әz!s Кpog

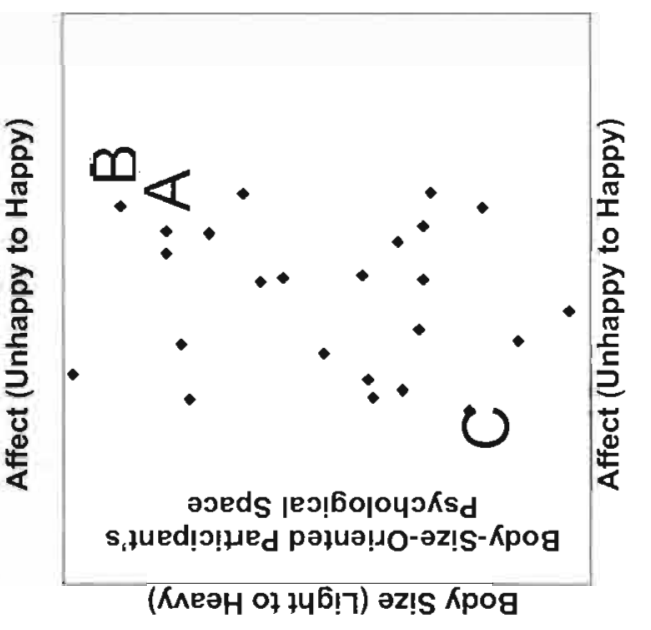

(К^еән 아 746 !) ) әZ!S Кров 
Conceptually, these attention weights stretch and shrink the $M$ dimensions of the group psychological space. The psychological space of a participant who attends much more to body size than to facial affect is presented in the lower left-hand panel of Figure 6.1. The large attention weight for body size increases the distance between the heavier and lighter photo stimuli, which reflects this participant's perception that heavier and lighter stimuli are very dissimilar to one another. In contrast, the small attention weight for affect shrinks the distance between the happier and sadder stimuli, consistent with the participant's judgment that happier and sadder stimuli are not particularly dissimilar. A particularly nice feature of the WMDS model is its simultaneous representation of both group- and participantspecific aspects of $\mathrm{PO}$ : Both the dimensions spanning the psychological space and the organization of the stimuli within each dimension are assumed to be shared by participants, whereas the relative attention to or importance of each dimension is allowed to vary across participants.

We have used the WMDS model to test hypotheses about clinically relevant individual differences in dimensional attention (Treat, McFall, Viken, \& Kruschke, 2001, 2006; Viken et al., 2002; for an alternative approach to the evaluation of dimensional attention, cf. chap. 4 , this volume). Within the realm of eating disorders, we have demonstrated that undergraduate women who report clinically significant symptoms of bulimia nervosa, compared with undergraduate women who report no bulimic symptoms, exhibit relatively greater attention to other women's body size than to their facial affect. Within the realm of sexual aggression, we have found that undergraduate men who display relatively greater attention to women's physical exposure (i.e., the extent to which the woman's clothing is revealing) than to women's facial affect (i.e., sad vs. happy) also construe the continuation of sexual advances toward an uninterested woman as more justifiable than men who attend relatively more to women's affect than exposure.

The WMDS model of participants' POs assumes that all participants organize the information within the dimensions to which they attend in the same way. Alternative MDS models of participants' POs, however, could be used to evaluate hypotheses about clinically relevant individual differences in the organization of stimuli within a dimension. For example, women who report eating-disorder symptoms might represent body-size information in a more discrete fashion as heavy or light, whereas control participants might perceive body size as varying more continuously. Evaluating this kind of research question typically would entail comparing the estimates of stimulus coordinates across individual-specific MDS solutions. Individualspecific MDS models estimate far more parameters than the WMDS model from the same amount of data, however. The resulting parameter estimates, 
therefore, likely will be very imprecise at the individual-participant level unless each participant provides more than one set of similarity ratings. Fortunately, Lee and Pope (2003) recently proposed a promising Bayesian model-fitting strategy that addresses these difficulties when analyzing all participants' data simultaneously by identifying subgroups of participants who share similar spatial representations of the stimuli and then estimating a separate MDS solution for each subgroup.

The WMDS model also assumes that participants perceive the stimulus dimensions to be uncorrelated (e.g., perception of body size is unrelated to perception of facial affect). A generalization of the WMDS model relaxes this constraint and allows investigators to characterize simultaneously both individual differences in dimensional attention and individual differences in the perceived correlation between dimensions (Tucker, 1972). It might be of interest, for example, to evaluate whether women with eating disorders, relative to control participants, perceive a stronger negative correlation between body size and affect, such that heavier women are perceived to be less happy and lighter women are perceived to be more happy. The same model could be used to evaluate whether sexually aggressive men perceive a stronger positive correlation between women's sexual interest and the provocativeness of women's dress than do control participants, whereby more provocatively dressed women are perceived to be more sexually interested and less provocatively dressed women are perceived to be less sexually interested.

All of the MDS models discussed to this point can be characterized as deterministic, because they model the location of stimuli in participants' psychological space as a single fixed point (i.e., stimulus values are assumed to be the same across trials). In contrast, probabilistic scaling approaches assume that the perceived value of the stimuli in psychological space fluctuates from trial to trial and represent stimulus locations as a multivariate normal distribution of values. MacKay and colleagues (MacKay \& Zinnes, 1986; Zinnes \& MacKay, 1992) have demonstrated that deterministic methods recover systematically biased estimates of stimulus coordinates whenever stimulus values are perceived variably, either across or within persons, and whenever stimuli are highly confusable. Under these circumstances, deterministic models tend to provide more extreme estimates of stimulus coordinates, such that high-variability stimuli are scaled toward the exterior of the psychological space (Zinnes \& MacKay, 1992). Treat et al, (2002) demonstrated the potential incremental utility of probabilistic methods under more clinically relevant conditions by juxtaposing deterministic and probabilistic solutions for men's perceptual organizations of women after the men had consumed and absorbed either an alcoholic or a placebo beverage. Deterministic and probabilistic solutions differed markedly for 
men in the alcohol condition, in particular, as would be expected given the greater perceptual variability presumably induced by alcohol consumption. In particular, dimensions were more difficult to interpret in the deterministic solutions, and the estimated configurations were characterized by a large empty space in the interior of the scaling solutions. Additionally, perceptual variability was significantly greater in the alcohol condition than in the placebo condition, and estimates of perceptual variability for each stimulus along the dimensions of "appeal" and "provocativeness" varied in a predictable fashion (e.g., seminude women were perceived homogeneously along the provocativeness dimension but heterogeneously along the appeal dimension).

Probabilistic MDS methods show clear incremental utility for mapping participants' psychological spaces whenever marked inter-or intraindividual perceptual variability either is assumed to be present or is of particular theoretical interest. These methods not only provide increased confidence in the accuracy of the estimated stimulus configuration but also estimate the magnitude of inter- or intraindividual perceptual variability, which also may interest clinical researchers. Additionally, probabilistic approaches allow much more statistically rigorous hypothesis testing about the ideal dimensionality for scaling solutions and the equivalence of stimulus coordinates or variances than typically is possible when using deterministic methods (Zinnes \& MacKay, 1992). The greater complexity of probabilistic models, however, places greater demands on the similarity-ratings data. As a result, currently available versions of probabilistic scaling methods do not estimate individual differences parameters, such as dimensional attention weights or interdimensional correlations. Additionally, replicated ratings are necessary for precise parameter estimation in individual-specific analyses.

In the subsequent sections of this chapter, we introduce a class of theoretical, measurement, and analytical models developed by cognitive scientists to account for classification, memory, and learning processes. These models account simultaneously for the operation of very different processes partly by positing a shared spatial representation of participants' perceptual organizations (Nosofsky, 1992b). Thus, using similarity-scaling models to map participants' psychological spaces not only allows clinical researchers to evaluate clinically relevant questions about participants' POs but also sets the stage for theoretically coherent and analytically rigorous investigations of research questions about other higher order cognitive processes.

\section{IMPLICIT CLASSIFICATION}

Implicit classification refers to the placement of stimuli into categories without experimenter instruction as to the stimulus characteristics on which 
to base classifications or feedback about the accuracy of classifications. Whereas identification entails the assignment of a different category label to each stimulus, classification involves the assignment of the same category label to multiple stimuli. Thus, classification involves treating different stimuli within the psychological space as functionally equivalent. When women are classified as "heavy" or "light," for example, the variation in body size that occurs within each category is ignored.

Individual differences in the basis for implicit classifications should be of interest to clinical researchers, because different behavioral responses to stimuli may be secondary to variation in partitioning of the psychological space. In a current ongoing study, for example, college-age men have classified undergraduate women depicted in photos as "likely to be responsive to sexual advances" or "not likely to be responsive to sexual advances." The women in the photos have varied orthogonally along two dimensions that may be relevant to men's classifications: (a) the provocativeness of the woman's clothing and (b) the woman's affect (i.e., the extent to which she is communicating strong sexual interest or strong sexual disinterest by means of her facial expression or body posture). In an alternative paradigm, the prototype-classification task, participants might classify stimuli as an example of one of two types of stimuli that vary along two orthogonal stimulus dimensions (Cohen \& Massaro, 1992). In the current study, one type of woman might be represented by a sexually interested and provocatively dressed woman, whereas the other type of woman might be represented by an angry woman who is not dressed provocatively. Either paradigm could be used to assess individual differences in college men's sensitivity or attention to these two dimensions when judging a woman's responsiveness, which may contribute to individual differences in the extent to which college men exhibit sexually aggressive behavior.

To date, we have relied primarily on the prototype-classification task to assess individual differences in participants' sensitivity or attention to underlying dimensions. In a recent study that examined the role of classification processes in eating disorders, participants first viewed a "Type A woman," who was normatively sad and heavy, and a "Type B woman," who was happy and light (Viken et al., 2002). See Figure 6.2 for an example of similar prototypes. Participants then classified each of the remaining stimuli as more similar to a Type A or a Type B woman. As when completing the similarity-ratings paradigm, participants were urged to respond quickly, because their first impressions were of primary interest to the experimenter.

According to Nosofsky's (1987) weighted prototype model, individual differences in participants' classification patterns partially reflect individual differences in participants' sensitivity or attention to the underlying dimensions. Suppose, for example, that a participant must classify a normatively sad and light woman, labeled stimulus $i$ in Figure 6.2. Classification of 


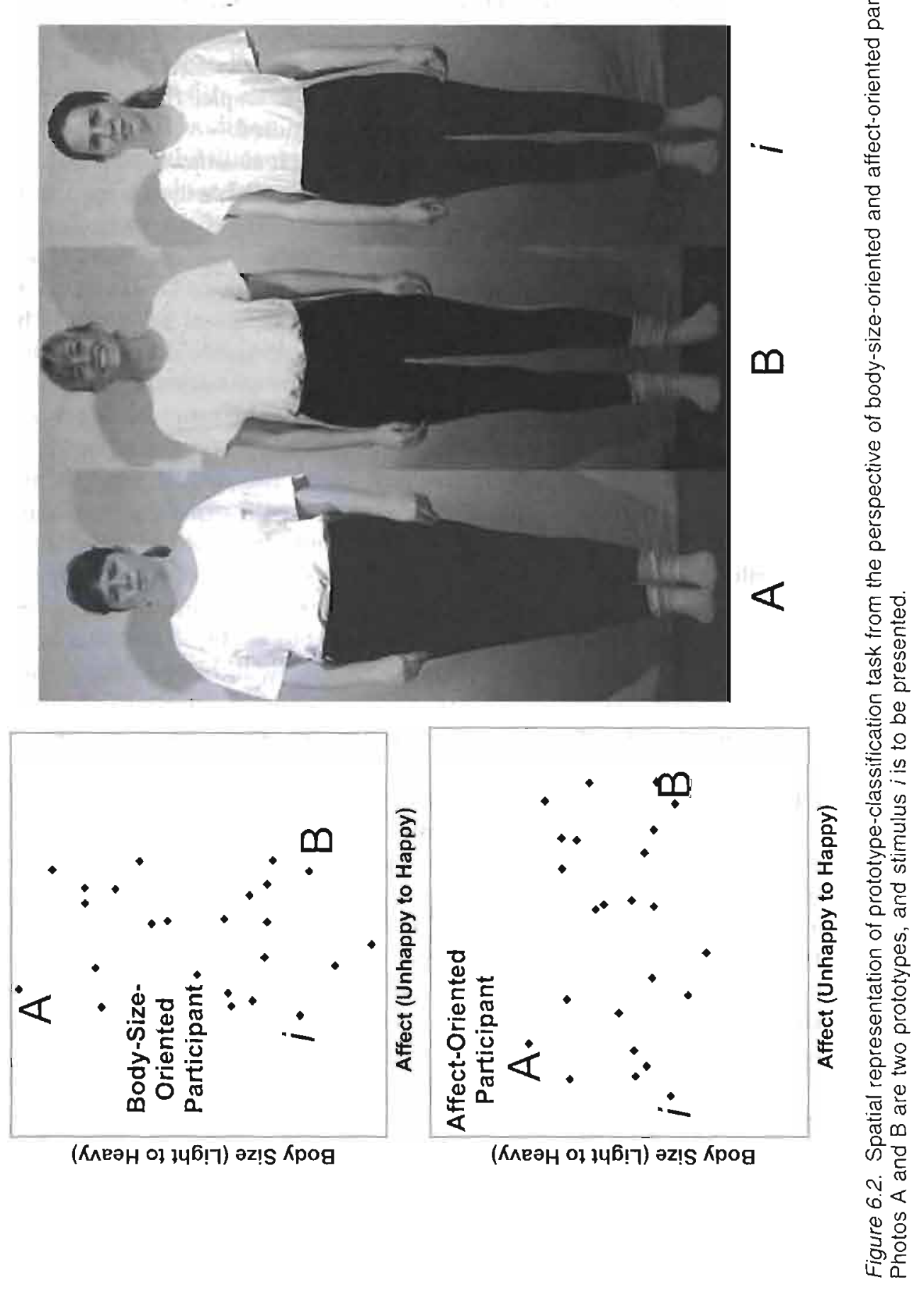

190 TREAT ET AL. 
stimulus $i$ as a Type A woman suggests greater attention to affect, whereas classification as a Type B woman suggests greater attention to body size. Put more formally, the probability of classifying stimulus $i$ as a member of Category $\mathrm{A}$, or $\mathrm{P}(\mathrm{A} \mid i)$ in Equation 6.3, is modeled as a function of the bias-weighted relative perceived similarity of stimulus $i$ to Prototypes $A$ and $\mathrm{B}$, or $\eta_{\mathrm{iA}}$ and $\eta_{\mathrm{B}}$ :

$$
P(A \mid i)=\frac{\beta_{A} \eta_{i A}}{\beta_{A} \eta_{i A}+\left(1-\beta_{A}\right) \eta_{i B}}, \eta_{i A}=e^{-c d i A}, d_{i A}=\left(\sum_{m=1}^{M} w_{m}\left(x_{i m}-x_{j m}\right)^{r}\right)^{1 / r} .
$$

Perceived similarity is assumed to be an exponentially decreasing function of the weighted distance between stimulus $i$ and the relevant prototype, or $-c d_{i A}$, where $c$ indicates the overall level of stimulus discriminability. As in the previous distance formula, $x_{i}$ and $x_{j}$ specify the stimulus coordinates, $M$ refers to the stimulus dimension, and $w_{m}$ indicates dimensional attention. Biases in the use of the category responses, $\beta_{\mathrm{A}}$ and $\beta_{\mathrm{B}}$, quantify participants' relative use of the two response categories. Thus, as illustrated in Figure 6.2, the probability of classifying stimulus $i$ as a member of Category A will be large when stimulus $i$ is perceived to be very similar to Prototype $A$ and very dissimilar to Prototype $B$, because the distance between stimulus $i$ and Protorype A will be much shorter than the distance between stimulus $i$ and Prototype $B$. If a participant also uses the two category responses at a similar rate (i.e., $\beta_{A}$ and $\beta_{B}$ have similar values), then $\mathrm{P}(\mathrm{A} \mid i$ ) will approach 1.0.

Viken et al. (2002) recently used Nosofsky's (1987) weighted prototype classification model to evaluate rigorously the hypothesis that a group of college women who reported clinically significant bulimic symptoms, relative to a group of control participants, would base their implicit classifications of other women relatively more on their body size than on their facial affect. In other words, we anticipated that a model specifying group-specific estimates of dimensional attention, or $w_{m}$, would fit bulimics' and control participants' classification patterns significantly better than a model that specified a shared estimate of dimensional attention across groups. The former model generalized the latter model (i.e., it adds a parameter to an otherwise identical set of parameters), so we were able to evaluate this hypothesis using nested model-comparison techniques (Wickens, 1989). As expected, the fit of the model to the data improved significantly when dimensional attention was assumed to be group specific but not when the remaining parameters were assumed to be group specific. Inspection of parameter estimates indicated that bulimic participants, compared with control participants, attended relatively more to body size than to facial affect.

The theoretical, measurement, and process models of implicit classification presented in this section could be used widely to examine clinically 
relevant questions about the role of classification processes in psychopathology. Group differences in dimensional attention estimates were of primary interest to Viken et al. (2002), because the implicit nature of the prototypeclassification task tended to elicit highly homogeneous use of the response categories across participants (i.e., response bias estimates were nearly identical for the two categories). Response bias estimates should exhibit much greater variability in an explicit prototype-classification task, however. Suppose, for example, that participants were instructed to base their classifications on the women's body size. In this case, group differences in dimensional attention should decrease sharply, because both groups would direct their attention to body size, but group differences in response bias estimates should reflect bulimic participants' less frequent use of the category exemplified by the thinner woman. Alternatively, we might examine individual differences in the extent to which men's explicit classifications of women's affect are influenced by the provocativeness of women's dress. The extent to which men attend to women's provocativeness, even when directed to attend to their affect, should increase the likelihood of their exhibiting sexually aggressive behavior. Overall, the models presented in this section provide a flexible approach to examination of a variety of questions about the role of implicit and explicit classification processes in psychopathology.

\section{RECOGNITION MEMORY}

Recognition memory refers to the observer's classification of stimuli as previously viewed or not. Cognitive scientists typically refer to this memory process as explicit, because participants are instructed to indicate whether they remember viewing the stimuli previously. Individual differences in explicit memory for stimulus features interest clinical researchers because such differences presumably contribute to clinically relevant variability in behavioral responses to stimuli. For example, college men who exhibit excellent memory for the provocativeness of women's dress but poor memory for women's affect should be at higher risk of exhibiting behavior that is judged to be sexually inappropriate or aggressive, because they are acting on the basis of incomplete or distorted information. Similarly, college women who struggle with problematic eating patterns may experience interpersonal difficulties or construe thinness as a royal road to happiness partially because they retain impoverished or erroneous information about the affect and happiness of other women.

Individual differences in explicit memory for stimulus attributes commonly are assessed in a recognition-memory task, in which participants first view, study, or are exposed to a subset of stimuli. Participants typically are not forewarned about the upcoming memory test or the relevant stimulus 
dimensions, but manipulations of these task features may be of theoretical interest to some researchers. At test, participants view both old stimuli and new stimuli that were not viewed previously. Participants classify each stimulus as old or new. Participants also may judge how confident they feel about these classifications. In a recently completed study (Treat, McFall, et al., 2006), college women first studied 28 photos of women who varied along body-size and facial-affect dimensions for 3 seconds apiece. Next, participants completed the prototype-classification task described above with the same 28 stimuli. Finally, participants viewed 56 photos of women and indicated whether they had seen the exact photo previously and how confident they were about their judgment by responding "definitely yes," "probably yes," "maybe yes," "maybe no," "probably no," or "definitely no." Half of the 56 photos were identical to those viewed previously; the remaining 28 photos showed the same woman, but either her affect or body size differed from the original (see Figure 6.3). Participants were told at the time of test that either "the look on her face" or "the shape of her body" might have been altered in each photo.

Signal-detection theory (SDT) methods (Macmillan \& Creelman, 1991) commonly are used to quantify individual differences in participant memory - that is, individual differences in participants' abilities to detect the old signal in the presence of either new-affect or new-body-size noise (for an alternative approach to modeling recall and recognition-memory data, see chap. 2, this volume). In the present case, we fic an SDT model to the observed frequencies of each participant's confidence ratings for old, new-affect, and new-body-size stimuli. Table 6.1 provides the observed data for a participant who displayed excellent memory for affect, as indicated by her correct classification of most of the old stimuli $(n=28)$ as definitely or probably seen previously, as well as her classification of most of the newaffect stimuli $(n=14)$ as definicely or probably nor seen previously. In contrast, her memory for body size was quite poor, as indicated by her inability to discriminate well between old and new body-size stimuli. The SDT model for recognition memory in this case, as illustrated in Figure 6.4 , assumes that the stimuli are arrayed along a dimension of perceived familiarity. The stimuli are members of either the old, the new-affect, or the new-body-size distributions, which are assumed to be normal in form. Exemplars from the old distribution presumably provoke a greater sense of familiarity on average, so this distribution is placed to the right of the other distributions in the figure. The new-affect distribution is placed to the left of the new-body-size distribution to reflect our expectation that memory will be greater for affect than for body-size information. Five boundaries, which are represented by dashed lines in the figure, partition the familiarity dimension into the six confidence rating categories. On a given trial, a stimulus is assumed to evoke a particular value along the familiarity 


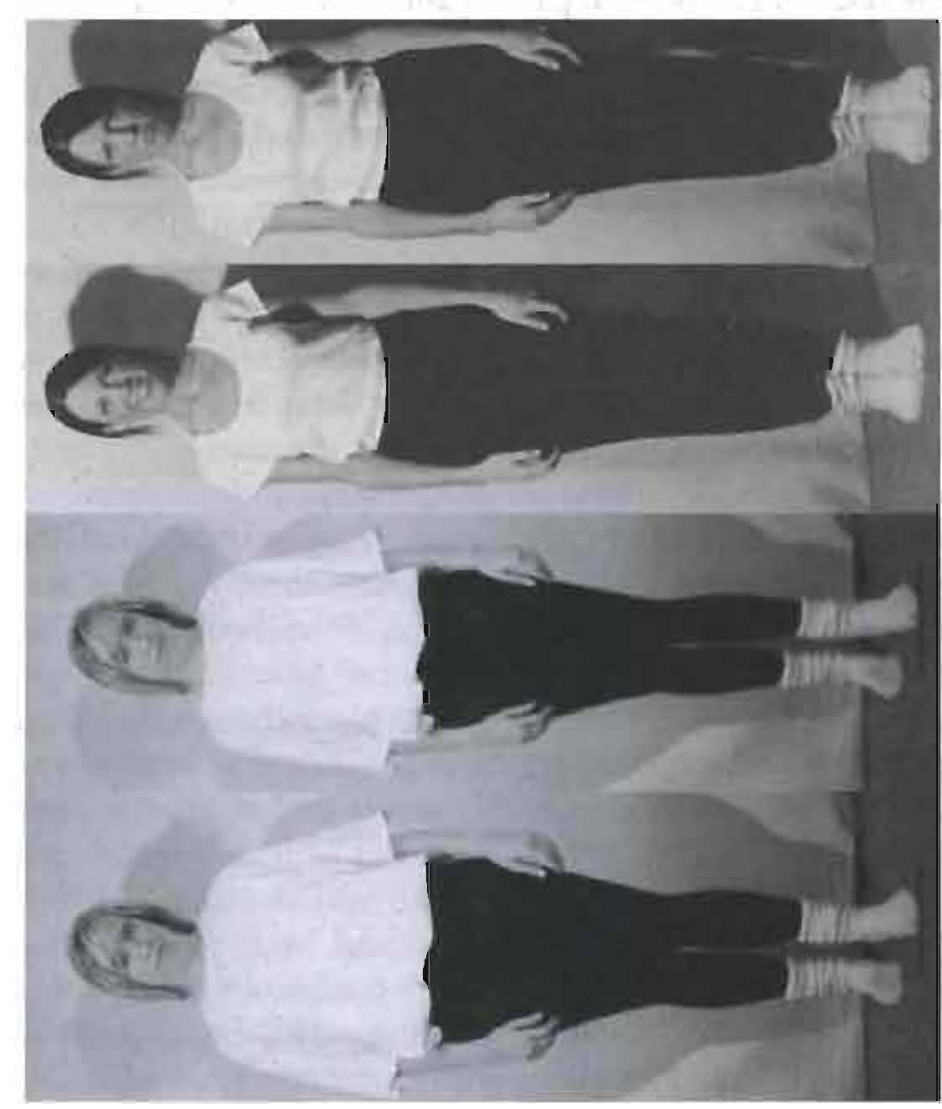

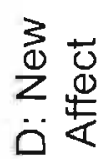

$\stackrel{\dot{m}}{\stackrel{5}{\leftrightarrows}}$

응

它

요



잉

范贺

盯

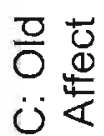

율

\&

它

ㅇํㄹ

공

(1) 3

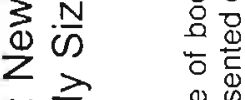

ஸ் 흥

응

क

등

N $\stackrel{0}{=}$

$O \frac{N}{O} \quad E=\frac{D}{Q}$

은

ष

4 ○

궁휴 $\frac{1}{\pi}$

$\stackrel{\square}{E} \cdot \frac{\pi}{0}$

(1)

$E \stackrel{0}{=}$

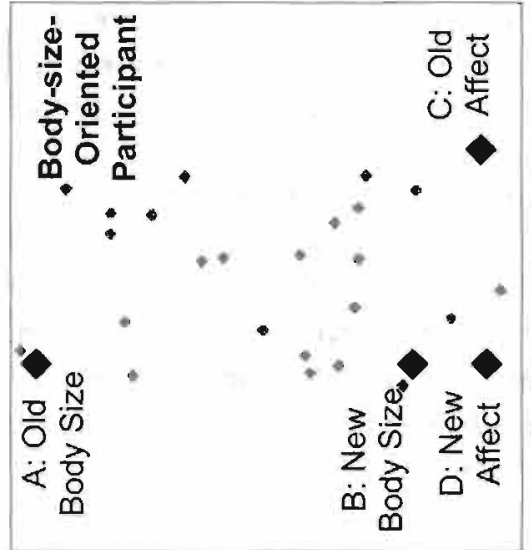

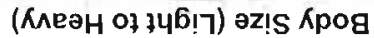

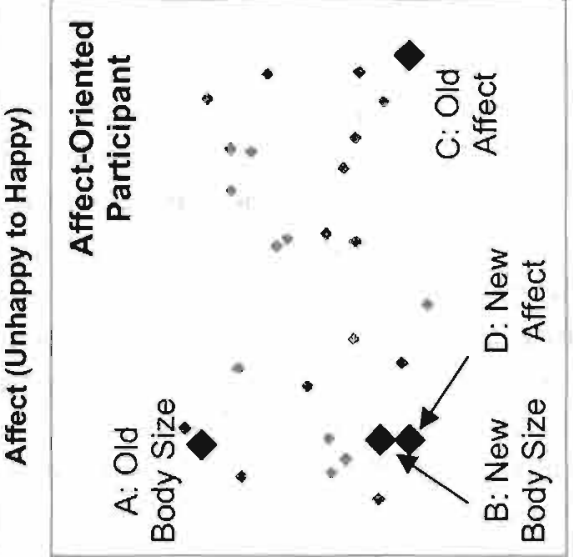

(Кмеән 아 146!า) әz!S Кров
동요

든 은

तิ

응 는

○용

드은 중 윻

$\div \geq \overline{0}$

के 응

屯웡 등

응 $\frac{0}{\pi}$

닌

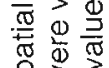
各 3 के लं क्ष 6 음응

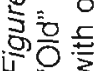


TABLE 6.1

Data Structure and Sample Frequency Data for Recognition-Memory Task

\begin{tabular}{lcccccc}
\hline & \multicolumn{6}{c}{$\begin{array}{c}\text { Participant response to "Have you seen this } \\
\text { EXACT PHOTO before?" }\end{array}$} \\
\cline { 2 - 8 } Photo classification & $\begin{array}{c}\text { Definitely } \\
\text { yes }\end{array}$ & $\begin{array}{c}\text { Probably } \\
\text { yes }\end{array}$ & $\begin{array}{c}\text { Maybe } \\
\text { yes }\end{array}$ & $\begin{array}{c}\text { Maybe } \\
\text { no }\end{array}$ & $\begin{array}{c}\text { Probably } \\
\text { no }\end{array}$ & $\begin{array}{c}\text { Definitely } \\
\text { no }\end{array}$ \\
\hline Old & 6 & 8 & 8 & 4 & 2 & 0 \\
New affect & 0 & 1 & 2 & 2 & 4 & 5 \\
New body size & 3 & 3 & 2 & 3 & 2 & 1 \\
\hline
\end{tabular}

dimension and then is classified into the category associated with that value of familiarity. For example, presentation of a previously viewed woman with a startlingly different facial expression might provoke an extremely low feeling of familiarity that does not clear even the lowest boundary between "definitely no" and "probably no." Hence, the participant would respond "definitely no."

Fitting the SDT model to each participant's frequency data entails using maximum-likelihood methods to estimate the means and standard deviations of the two new distributions, because the mean and standard

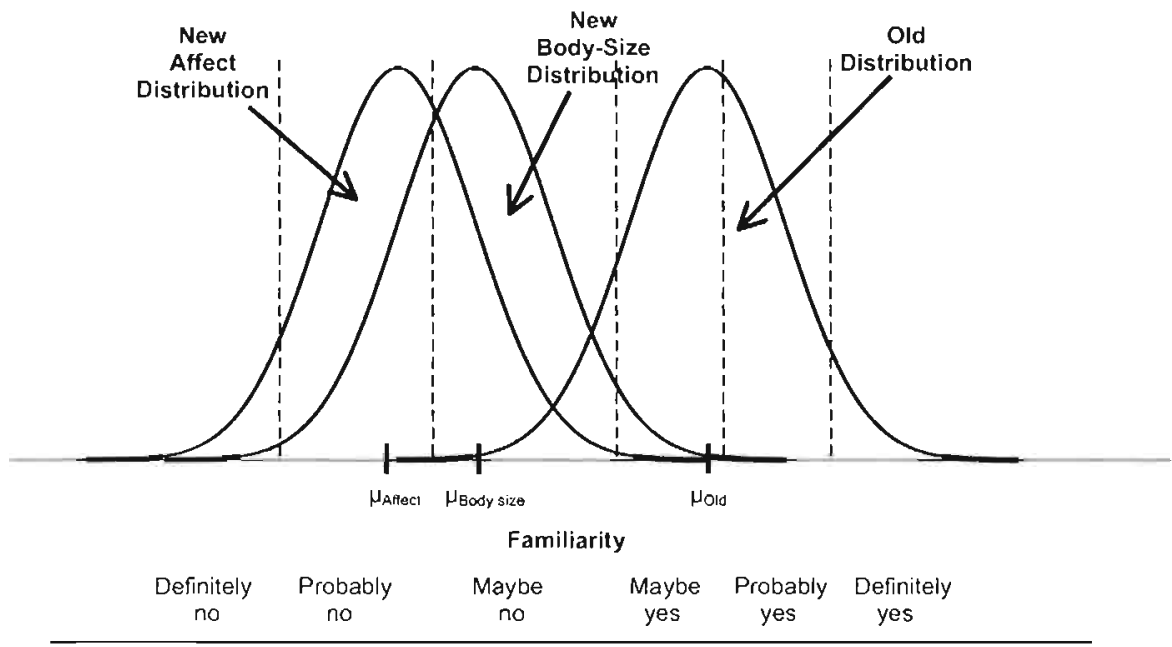

Confidence Rating

Figure 6.4. Signal-detection theory model for recognition-memory task, in which participants judge how confident they are that they have seen the exact photo previously. Stimuli from the "old" distribution were viewed by participants prior to the memory task; stimuli from the "new" distributions portray the same women with opposing values along either the body-size or facial-affect dimensions and are assumed to evoke a weaker feeling of familiarity. See text for further details. 
deviation of the old distribution can be fixed at 0.0 and 1.0 , respectively. The locations of the five boundaries separating the confidence regions also are estimated. Given these parameter estimates, the standardized distance between the mean of the old distribution and the mean of one of the new distributions indicates the strength of a participant's memory for either affect or body-size information. In spite of the sparseness of individual participants' data and the relatively large number of estimated parameters, individual differences in memory for body size and affect correlated reliably with women's self-reported symptoms of eating disorders. Relative to control participants, college women who reported clinically significant symptoms of eating disorders showed significantly and substantially impaired memory for affect information, consistent with their markedly decreased attention to affect in the prototype-classification task. In contrast, control participants and high-symptom participants showed similar levels of memory for body-size information, even though high-symptom participants showed significantly greater attention to body-size information than did control participants in the prototype-classification task. This finding suggests that future research should explore whether high-symptom participants, relative to low-symptom participants, store or retrieve body-size information differently or show decreased attention to body-size information during the recognitionmemory task.

The SDT model is a commonly used process model of recognition memory in clinical research, as it specifies the decision-making processes that operate on the feeling of familiarity evoked by each stimulus to produce participant responses. The SDT model does not specify the representational and memory processes that give rise to the feeling of familiarity, however. In contrast, Nosofsky's (1991, 1992a, 1992b) process model of recognition memory emphasizes the central role of stimulus representation and memory processes in producing participant perceptions of stimulus familiarity, or $F_{i}$ in Equation 6.4. Nosofsky's model operates on the stimulus similarities implied by the participant's psychological space and formalizes the notion that a stimulus presented at test, $i$, will tend to be recognized as old when its summed similarity to all $J$ stimuli previously stored in memory, $F_{i}$, clears an estimated threshold. In the memory model in Equation 6.4, $\mathrm{S}_{j}$ refers to the strength with which stimulus $j$ is stored in memory. As in the classification model described above, the similarity of the presented stimulus to each stored stimulus, $\eta_{i j}$, is modeled as an exponentially decreasing function of the dimensionally weighted distance between the two stimuli, $d_{i j}$ :

$$
F_{i}=\sum_{j=1}^{J} S_{j} \eta_{i j}, \eta_{i j}=e^{-c d i j}, d_{i j}=\left(\sum_{m=1}^{M} w_{m}\left(x_{i m}-x_{j m}\right)^{r}\right)^{1 / r} .
$$


Figure 6.3 illustrates the psychological space of the previously stored stimuli as well as the location of two new stimuli for both body-size and affect-oriented participants. Note that the Euclidean distance between the relevant old and the new stimulus-or the perceived dissimilarity of the relevant old and the new stimulus-varies markedly for these 2 participants. In particular, the distance between these stimuli is much greater when the altered dimension is the focus of attention for the participants (e.g., the distance between old-and new-affect stimuli is much greater for affectoriented participants than for body-size-oriented participants). According to Equation 6.4, this increased distance translates into decreased similarity, which leads to a feeling of decreased familiarity, other things being equal. Thus, a body-size-oriented participant should perceive new affect stimuli to be more familiar than an affect-oriented participant. We anticipate that when we fit this model to our recently collected memory data, we should be able to account for the marked group differences in memory for affect by allowing $w_{\text {affec }}$ to be significantly smaller and different for the women who report clinically significant symptoms of eating disorders. In a similar study examining individual differences in men's perceptions of women, we anticipate that men who exhibit sexually aggressive behavior will show deficient memory for women's affect that can be attributed to their significantly reduced attention to women's affect.

Clinical scientists could use the process model of recognition memory described above to address a wide variety of research questions about the role of explicit memory processes in psychopathology. To date, we have focused on the role of dimensional attention in accounting for group differences in recognition memory. Research questions also might focus on clinically relevant variability in other parameters, however, such as the estimated cutoffs for old-new or confidence-rating judgments, which capture individual differences in how conservatively or liberally participants use the response categories, and the memory strengths for individual stimuli or for stimuli in particular regions of the psychological space, which represent individual differences in the strength with which stimuli are stored in memory. Additionally, clinical researchers may be interested in the impact of various instructional manipulations on memory processes, as they move researchers in the direction of considering how to ameliorate problematic memory deficits. In the described study, participants were not told anything about the relevant stimulus attributes or the upcoming memory task. Future research should determine which (if any) of the following three manipulations improve high-symptom women's memory for affect substantially: (a) noting that the stimuli vary along affect and body-size dimensions before having participants study the stimuli; (b) alerting participants before they study the stimuli about the existence of an upcoming memory task for unnamed 
stimulus features; or (c) directing participants' attention, before they familiarize themselves with the stimuli, to the two relevant stimulus attributes for which memory will be assessed later. Thus, the theoretical, measurement, and analytical models presented in this section can be used to evaluate a plethora of research questions about the role of recognition memory processes in psychopathology.

\section{CATEGORY LEARNING}

In contrast to implicit classification, category learning refers to the placement of stimuli into categories with feedback about the accuracy of classification, although experimenter instruction about the stimulus characteristics on which to base classifications typically remains absent. Evaluating individual differences in category learning about clinically relevant information should prove to be of particular interest to clinical researchers, for several reasons. First, this approach facilitates investigations of individual differences in more dynamic aspects of cognitive processing, which may afford incremental prediction of clinical difficulties above and beyond more static characterizations of processing. For example, Viken et al. (2002) demonstrated previously that body-size-oriented women, who exhibit relatively greater attention to other women's body size than to facial affect, are more likely to report clinically significant symptoms of eating disorders. Body-size-oriented women presumably would exhibit variability in how rapidly they learn a simple affect category structure, however, in which happy women are in one category and sad women are in another. In this case, individual differences in how rapidly participants could learn an affect category structure might be more diagnostic of eating difficulties than individual differences in their pre-existing perceptual organizations.

Second, fitting formal process models of category learning to participants' observed data (e.g., their trial-by-trial responses) affords examination of the relative importance of various mechanisms that are hypothesized to underlie learning. Investigation of the processes underlying learning ultimately should facilitate the development of more targeted and efficient treatment approaches. Finally, category-learning paradigms may provide an alternative approach to cognitive therapy that draws on experimental cognitive psychology to retrain or modify deficient cognitive processing. Current cognitively oriented treatments rely on verbally mediated techniques that emphasize the identification and modification of specific maladaptive thoughts and beliefs. Using the tools of contemporary cognitive science, we may be able to develop performance-based interventions that target specific deficits in cognitive processing. For example, a new form of 
cognitive therapy might entail using learning paradigms to retrain attentional patterns, speed the gradual acquisition of relevant category structures, or facilitate attention shifting to relevant stimulus features under highrisk conditions.

Treat et al. (2001) illustrated the use of a category-learning task to assess individual differences in men's cognitive processing of the physical appearance and perceived affect of women. A stimulus set containing 26 photos of women from newsstand magazines varied along two dimensions of primary theoretical interest: women's physical exposure and women's facial affect. College men viewed one photo at a time, classified it as a member of one of two categories with arbitrary labels (e.g., "Category F" and "Category J"), and received feedback on the accuracy of their classifications (e.g., "Correct! She is a member of Category J"). Participants were told that initially they would be guessing, and they were not told the basis for the feedback. Participants also were told that the basis for the feedback might change during the course of the task and that they should attempt to learn the new category labels for the stimuli if this occurred. Participants first completed four blocks of either an exposure or affect category structure. In the affect category structure, for example, women exhibiting positive affect were in Category $F$, and women exhibiting negative affect were in Category J. After an unannounced shift to the other category structure, participants completed an additional four blocks of training. Before the category-learning task, participants had completed a similarity-ratings task with a subset of the stimuli, which allowed the experimenters to classify participants' perceptual organizations as either exposure oriented or affect oriented. As expected, exposure-oriented participants performed better at learning the exposure category structure than did affect-oriented participants, whereas affect-oriented participants performed better at learning the affect category structure. In other words, participants learned a category structure much more rapidly when it was congruent with their underlying perceptual organization.

Fitting formal process models of category learning to participants' observed data (e.g., their trial-by-trial responses) affords examination of the relative importance of various mechanisms that are hypothesized to underlie participants' observed responses. To date, we have relied on process models developed by Kruschke and colleagues (Kruschke, 1992; Kruschke $\&$ Johansen, 1999), because they also treat a spatial representation of participants' psychological space as the primitive, as do Nosofsky's (1987, 1991, 1992a, 1992b) prototype-classification and memory models. We fit Kruschke and Johansen's (1999) "rapid attention shifts 'n' learning" (RASHNL) model to the learning data described above, so that we could evaluate the importance of three potential influences on participants' learning and group 
differences in participants' learning (for explication of another formal quantitative approach to examination of category-learning processes, see chap. 4 , this volume).

The first mechanism of interest, initial differences in relative attention to the psychological dimensions of physical exposure and facial affect, may facilitate or inhibit learning a particular category structure, depending on their congruence with the structure to be learned. For example, affectoriented participants should be at a relative advantage over exposureoriented participants when learning the affect category structure, because they perceive stimuli in the same category to be relatively more similar than stimuli in different categories. The second mechanism, shifting attention toward relevant dimensions and away from irrelevant dimensions, suggests that participants learn category structures by modifying their perceptual organization to be more consistent with the demands of the category structure. In other words, exposure-oriented participants could learn the affect category structure by increasing their attention to affect and decreasing their attention to exposure, thus modifying their perceptual organization to make it similar to that of the affect-oriented participant. This shift in dimensional attention would enhance the relatively greater perceived similarity of stimuli in the same category to stimuli in different categories. The third mechanism attributes participant learning to strengthened associations between regions of the psychological space and correct category responses. Participants could learn the affect category structure, for example, by gradually mapping the region of the psychological space that contains women displaying positive affect to the "F" response and the region of the psychological space that contains women exhibiting negative affect to the "J" response.

We fit the RASHNL model (Kruschke \& Johansen, 1999) to the proportion-correct values of the exposure- and affect-oriented groups on each of the eight blocks in the learning task to evaluate the relative importance of these three mechanisms to participants' learning. Preliminary model fits indicated that participants perceived the stimulus dimensions in a holistic rather than a separable fashion, so the Euclidean metric was used to define interstimulus distances in the remaining analyses. As expected, the bestfitting RASHNL model necessitated retention of a group-specific estimate of initial differences in relative attention, with exposure-oriented participants showing significantly greater relative attention to exposure than to affect compared with the affect-oriented participants. This finding is consistent with the results of the initial statistical analyses of the observed data that we described above; however, fitting a process model to the data also provided group-specific estimates of relative attention to the psychological dimensions of interest.

Although extensive evidence supports a role for attention shifting in category learning within cognitive science, the best-fitting RASHNL model 
suggested that shifting attention toward relevant dimensions and away from irrelevant dimensions did not play a role in participants' learning. The "stickiness" of participants' perceptual organizations may be attributable in part to participants' more holistic processing of the stimulus dimensions, which increases the difficulty of shifting attention toward or away from specific dimensions (Nosofsky \& Palmeri, 1996). Finally, the RASHNL modeling indicated that learning to map regions of the psychological space to the correct category label played a central but not group-specific role in participants' acquisition of the category structures. This association-learning mechanism produces more gradual, incremental improvement in performance than the attention-shifting mechanism.

The ability to shift attention to newly relevant stimulus information is highly adaptive under some conditions, such as when a person needs to shift attention away from a potential sexual partner's physical exposure or sexual characteristics and toward the partner's expressions of sexual interest. Thus, it may prove fruitful to develop novel treatment strategies that use category-learning paradigms therapeutically to modify problematic attention patterns. The present findings are somewhat discouraging in this regard, because the RASHNL model fits suggested that participants did not learn by optimizing their dimensional attention patterns. Participants' more integral processing of the stimulus dimensions presumably rendered attention shifting more difficult, but this will be a commonly occurring feature of the more complex stimulus sets of interest to clinical researchers. Thus, future research should investigate the learning conditions under which participants' perceptual organizations exhibit greater flexibility and malleability, even when the stimulus dimensions are perceived more holistically.

This extended presentation of Treat et al.'s (2001) category-learning study was intended to illustrate the potential utility of investigating clinically relevant category-learning processes as well as using formal process models to characterize the operation of mechanisms hypothesized to underlie both normal and abnormal learning patterns. This study relied on a relatively simple category-learning task, in which each category structure was based on a single dimension and a single unannounced shift to a new category structure occurred, but more complex associative-learning paradigms (e.g., forward-and backward-blocking paradigms, learned-inhibition paradigms) also should prove useful to clinical researchers. In a recently completed study using a more complex paradigm, for example, Treat, Kruschke, and McFall (2006) demonstrated that learning a category structure based on an initially irrelevant dimension is strikingly more difficult after learning a category structure based on an initially relevant dimension. In this study, a significant subgroup of body-size-oriented women were unable to learn an affect category structure (happy vs. sad) after learning a body-size category structure (heavy vs. light), although all body-size-oriented women were able 
to learn an affect category structure when it was presented first. Future research will examine whether women who struggle with eating-disorder symptoms are overrepresented in this subgroup, because deficient processing of affective information may help to account for the marked interpersonal and emotion-regulation difficulties that frequently characterize this population.

Additionally, Treat et al.'s (2001) study provided deterministic feedback to participants (i.e., each stimulus always received the same feedback within a particular category structure). The administration of probabilistic feedback (e.g., each stimulus might receive the same feedback $80 \%$ of the time) provides a much more externally valid approximation of real-world feedback, such as women's responses to sexual advances or women's feelings about themselves, which rarely are perfectly consistent across occasions. Thus, it should prove useful to characterize individual differences in participants' learning about clinically relevant information under more challenging probabilistic conditions.

\section{CONCLUSION}

In this chapter, we have provided an overview of an integrated class of cognitive science models that can be used to conceptualize, measure, and model clinically relevant individual differences in cognitive processing. The process models of classification, memory, and learning account simultaneously for performance on very different tasks by positing a shared representational substrate, which can be assessed using MDS methods (Nosofsky, 1992b). Thus, these models display a high degree of coherence and consistency across their theoretical, measurement, and analytical layers. Additionally, they afford examination of the mechanisms hypothesized to underlie observed task performance, which provides researchers with a much richer understanding of individual differences in the operation of the cognitiveprocessing system.

Several studies now have demonstrated the feasibility and utility of adopting this quantitative approach to clinical cognitive science, in which clinical scientists represent clinically relevant individual differences in cognitive processing within cognitive scientists' formal computational models by modifying parameter values or architectures (e.g., Busemeyer \& Stout, 2002; Filoteo \& Maddox, 1999; Neufeld et al., 2002; Treat et al., 2001, 2002; Viken et al., 2002). These studies highlight the generalizability of cognitive science theories, methods, and process models to more real-world circumstances, in which individual differences in the processing of socially complex information are the phenomenon of interest. These quantitative modeling efforts should advance clinical scientists' theories about cognitive 
influences on psychopathology, suggest novel intervention targets, and foster the development of novel forms of performance-based cognitive therapy.

\section{REFERENCES}

Beck, A. T. (1976). Cognitive theory and the emotional disorders. New York: International Universities Press.

Busemeyer, J. R., \& Stout, J. D. (2002). A contribution of cognitive decision models to clinical assessment: Decomposing performance on the Bechara gambling task. Psychological Assessment, 14, 253-262.

Carroll, J. D., \& Chang, J. J. (1970). Analysis of individual differences in multidimensional scaling via an $N$-way generalization of "Eckart-Young" decomposition. Psychometrika, 35, 283-320.

Cohen, M. M., \& Massaro, D. W. (1992). On the similarity of categorization models. In F. G. Ashby (Ed.), Multidimensional models of perception and cognition (pp. 395-447). Hillsdale, N]: Erlbaum.

Davison, M. L. (1992). Multidimensional scaling. Malabar, FL: Krieger.

Filoteo, J. V., \& Maddox, W. T. (1999), Quantitative modeling of visual attention processes in patients with Parkinson's disease: Effects of stimulus integrality on selective attention and dimensional integration. Neuropsychology, 13, 206-222.

Kelly, G. A. (1955). The psychology of personal constructs. New York: Norton.

Knight, R. A., \& Silverstein, S. M. (2001). A process-oriented approach for averting confounds resulting from general performance deficiencies in schizophrenia. Joumal of Abnormal Psychology, 110, 15-30.

Kruschke, J. K. (1992). ALCOVE: An exemplar-based connectionist model of category learning. Psychological Review, 99, 22-44.

Kruschke, J. K., \& Johansen, M. K. (1999). A model of probabilistic category learning. Joumal of Experimental Psychology: Learning, Memory, and Cognition, $25,1083-1119$.

Lee, M. D., \& Pope, K. J. (2003). Avoiding the dangers of averaging across subjects when using multidimensional scaling. Joumal of Mathematical Psychology, 47, $32-46$.

MacKay, D. B., \& Zinnes, ]. L. (1986). A probabilistic model for the multidimensional scaling of proximity and preference data. Marketing Science, 5, 325-344.

MacLeod, C. (1993). Cognition in clinical psychology: Measures, methods or models? Behaviour Change, 10, 169-195.

Macmillan, N. A., \& Creelman, C. D. (1991). Detection theory: A user's guide. Cambridge, England: Cambridge University Press.

McFall, R. M. (1990). The enhancement of social skills: An information-processing analysis. In W. L. Marshall \& D. R. Laws (Eds.), Handbook of sexual assault: Issues, theories, and treatment of the offender (pp. 311-330). New York: Plenum Press. 
McFall, R. M., \& Townsend, ]. T. (1998). Foundations of psychological assessment: Implications for cognitive assessment in clinical science. Psychological Assessment, 10, 316-330.

McFall, R. M., Treat, T. A., \& Viken, R. J. (1998). Contemporary cognitive approaches to studying clinical problems. In D. K. Routh \& R. J. DeRubeis (Eds.), The science of clinical psychology: Accomplishments and future directions (pp. 163-197). Washington, DC: American Psychological Association.

Neufeld, R. W. J., Vollick, D., Carter, J. R., Boksman, K., \& Jetté, ]. (2002). Application of stochastic modeling to the assessment of group and individual differences in cognitive functioning. Psychological Assessment, 14, 279-298.

Nosofsky, R. M. (1987). Attention and learning processes in the identification and categorization of integral stimuli. Journal of Experimental Psychology: Learning, Memony, and Cognition, 13, 87-108.

Nosofsky, R. M. (1991). Tests of an exemplar model for relating perceptual classification and recognition memory. Joumal of Experimental Psychology: Human Perception and Performance, 17, 3-27.

Nosofsky, R. M. (1992a). Exemplar-based approach to relating categorization, identification, and recognition. In F. G. Ashby (Ed.), Multidimensional models of perception and cognition (pp. 363-393). Hillsdale, N]: Erlbaum.

Nosofsky, R. M. (1992b). Similarity scaling and cognitive process models. Annual Review of Psychology, 43, 25-53.

Nosofsky, R. M., \& Palmeri, T. J. (1996). Learning to classify integral-dimension stimuli. Psychonomic Bulletin \& Review, 3, 222-226.

Schewe, P., \& O'Donohue, W. (1993). Rape prevention: Methodological problems and new directions. Clinical Psychology Review, 13, 667-682.

Shepard, R. N. (1964). Attention and the metric structure of the stimulus space. Journal of Mathematical Psychology, L, 54-87.

Treat, T. A., Kruschke, J. K., \& McFall, R. M. (2006). Individual differences in blocking of socially relevant information. Manuscript in preparation.

Treat, T. A., McFall, R. M., Viken, R. ]., \& Kruschke, ]. K. (2001). Using cognitive science methods to assess the role of social information processing in sexually coercive behavior. Psychological Assessment, 13, 549-565.

Treat, T. A., McFall, R. M., Viken, R. J., \& Kruschke, J. K. (2006). The role of attention, memory, and correlation-detection processes in eating disorders. Manuscript in preparation.

Treat, T. A., McFall, R. M., Viken, R. J., Nosofsky, R. M., MacKay, D. B., \& Kruschke, J. K. (2002). Assessing clinically relevant perceptual organization with multidimensional scaling techniques. Psychological Assessment, 14, 239252.

Tucker, L. R. (1972). Relations between multidimensional scaling and three-mode factor analysis. Psychometrika, 37, 3-27. 
Viken, R. J., Treat, T. A., Nosofsky, R. M., McFall, R. M., \& Palmeri, T. (2002). Bulimics and controls' differential attention to and classification of body-size and affect stimulus information. Journal of Abnormal Psychology, 111, 598-609.

Vitousek, K. B. (1996). The current status of cognitive-behavioral models of anorexia nervosa and bulimia nervosa. In P. M. Salkovskis (Ed.), Frontiers of cognitive therapy (pp. 383-418). New York: Guilford Press.

Ward, T., Hudson, S. M., Johnston, L., \& Marshall, W. L. (1997). Cognitive distortions in sex offenders: An integrative review. Clinical Psychology Review, 17, 479-507.

Wickens, T. D. (1989). Muliway contingency tables analysis for the social sciences. Hillsdale, NJ: Erlbaum.

Wilson, G. T. (1999). Cognitive behavior therapy for eating disorders: Progress and problems. Behaviour Research and Therapy, 37, S79-S95.

Zinnes, J. L., \& MacKay, D. B. (1992). A probabilistic multidimensional scaling approach: Properties and procedures. In F. G. Ashby (Ed.), Multidimensional models of perception and cognition (pp. 35-60). Hillsdale, NJ: Erlbaum. 


\title{
A D V A N C ES IN Clinical Cognitive Science
}

Formal Modeling of Processes and Symptoms

\author{
E D I T E \\ RICHARD W.J. NEUFELD
}

A MERICAN PSYCHOLOGICAL ASSOCIATION W A S H I G T O N, D C 
Copyright (c) 2007 by the American Psychological Association. All rights reserved. Except as permitted under the United States Copyright Act of 1976, no part of this publication may be reproduced or distributed in any form or by any means, including, but not limited to, the process of scanning and digitization, or stored in a database or retrieval system, without the prior written permission of the publisher.

Published by

American Psychological Association

750 First Street, NE

Washington, DC 20002

www.apa.org

To order

APA Order Department

P.O. Box 92984

Washington, DC 20090-2984

Tel: (800) 374.2721

Direct: (202) 336-5510

Fax: (202) 336.5502

TDD/TTY: (202) 336-6123

Online: www.apa.org/books/

E-mail: order@apa.org
In the U.K., Europe, Africa, and the Middle East, copies may be ordered from

American Psychological Association

3 Henrietta Street

Covent Garden, London

WC2E 8LU England

Typeset in Goudy by World Composition Services, Inc., Sterling, VA

Printer: Edwards Brothers, Ann Arbor, Ml

Cover Designer: Berg Design, Albany, NY

Technical/Production Editor: Harriet Kaplan

The opinions and statements published are the responsibility of the authors, and such opinions and statements do not necessarily tepresent the policies of the American Psychological Association.

\section{Library of Congress Cataloging-in-Publication Data}

Advances in clinical cognitive science : formal modeling of processes and symptoms / edited by Richard W. J. Neufeld.--1st ed.

p. ; cm.

Includes bibliographical references and index.

ISBN-13: 978-1.59147-784-6

ISBN-10: 1.59147 .784 .0

1. Psychology, Pathological. 2. Cognicive science. I. Neufeld, Richard W. J.

[DNLM: 1. Cognition Disorders-diagnosis. 2. Clinical Medicine-methods. 3. Cognition.

4. Cognitive Science-methods. 5. Models, Theoretical. WM 204 A244 2007]

RC454.4.A38 2007

$616.89075-\mathrm{dc} 222006023460$

British Library Cataloguing-in-Publication Data

A ClP record is available from the British Library.

Printed in the United States of America

First Edition 